DOI: 10.12731/2227-930X-2021-11-3-35-44 УДК 681.3

\title{
РАЗРАБОТКА АЛГОРИТМА АНАЛИЗА ПАРАМЕТРОВ В СПУТНИКОВОЙ СИСТЕМЕ СВЯЗИ
}

\author{
Мельникова Т.В., Преображенский А.П., \\ Преображенский Ю.П.
}

Спутниковые системы связи характеризуются большим числом параметров. Это определяет необходимость в выборе соответствуюших методов анализа, для того, чтобы обеспечить эффективную работу. Среди рассматриваемых признаков мы выделяем такие, которые группируются в определенные подмножества. Показано, как может сформироваться соответствующий критерий оптимальности в спутниковых системах, продемонстрировано, каким образом он будет учитьваться при создании алгоритма. Формируется случайная величина по завершению разбиения по подмножествам. Требуется получить оптимальный набор факторов. Они получаются из условия максимального значения выражения, представляющего собой сумму парных коэффищиентов корреляции. Указаны подмножества показателей, являющихся взаимосвязанными. Затем применяем методологию, связанную с построением «деревьев решений» для того, чтобы была сформирована модель дифференциального анализа. Дерево решений позволяет определить классы признаков. Число таких классов может быть более двух. В дереве решений выбираются линейные решающие функции последовательным способом. Они будут связаны с соответствующими критериями.

Ключевые слова: информация; управление; спутниковая система; параметр

\section{THE DEVELOPMENT OF AN ALGORITHM FOR ANALYSIS OF PARAMETERS IN A SATELLITE COMMUNICATION SYSTEM}

\section{Melnikova T.V., Preobrazhenskiy A.P., Preobrazhenskiy Yu.P.}

Satellite communication systems are characterized by a large number of parameters. This determines the need for the selection of appro- 
priate analysis methods in order to ensure effective operation. Among the features under consideration, we single out those that are grouped into certain subsets. It is shown how the corresponding criterion of optimality can be formed in satellite systems, it is demonstrated how it will be taken into account when creating an algorithm. A random variable is generated upon completion of subsetting. It is required to obtain an optimal set of factors. They are obtained from the condition of the maximum value of the expression, which is the sum of the pairwise correlation coefficients. The subsets of indicators that are interrelated are indicated. Then we apply the methodology associated with the construction of "decision trees" in order to form a model of differential analysis. The decision tree allows you to define the classes of characteristics. The number of such classes can be more than two. Linear decision functions are selected in the decision tree in a sequential manner. They will be linked to matching criteria.

Keywords: information; control; satellite system; parameter

\section{Введение}

В настоящее время можно наблюдать развитие систем, связанных с поддержкой принятия решений в спутниковых системах связи. Они базируются на том, что параметры, описывающие спутниковую систему связи [1], разбиваются по определенным группам с точки зрения их значимости. Анализ спутниковой системы связи должен базироваться на дифференциальном подходе. В этой связи представляет с точки зрения практики интерес разработка соответствующего алгоритма обработки параметров.

Цель работы заключается в рассмотрении возможностей построения алгоритма для анализа параметров в спутниковой системе связи.

\section{Алгоритм анализа значимости параметров в спутниковой} системе связи. Во многих случаях, когда анализируются спутниковые системы связи, тогда некоторые общие факторы в них могут меняться. Это может оказывать разное влияние на тех признаках, которые измеряются [2]. Например, по исходному множеству из $p$ 
признаков можно увидеть, что происходит процесс разбиения по (с p) относительно небольшому числу подмножеств. Анализ показывает, что изменение признаков, которые связаны с определенным подмножеством, базируется большей частью на каком-то одном общем факторе. Он будет своим по каждому из подмножеств. Примем такую гипотезу. Тогда будем разбивать по подмножествам так, чтобы параметры, которые лежат в определенном подмножестве, были коррелированны весьма сильным образом. При этом для параметров в разных подмножествах будет достаточно слабая. Формируем случайную величину по завершению разбиения по подмножествам [3].Она будет достаточно сильным способом коррелированна с параметрами такого подмножества. Интерпретируем такую случайную величину в виде искомого фактора. Он заметным образом будет влиять на все параметры в указанном подмножестве.

Если рассматривать общую логическую схему факторного анализа, тогда мы в ней выделяем какой-то частный случай. Для эвристически оптимизационного подхода [4] есть отличия от классических моделей в факторном анализе. Признаки группируются и общие факторы выделяются, базируясь на принципах экстремизации по определенным эвристическим способом обозначенных функционалов. Экстремальная группировка параметров [5] рассматривается в виде разбиений, ведущих к оптимизации функционала $J_{1}$ или $J_{2}$. Наборы случайных величин $x^{(1)}, x^{(2)}, \ldots, x^{(p)}$ будут группироваться экстремальным образом по предварительно указанному количеству классов $p$ с точки зрения поиска соответстствующих подмножеств $S_{1}, S_{2}, \ldots S_{p}$. При этом числа натуральные $1,2, \ldots p$, что $\cup_{l=1}^{p} S_{l}=\{1,2, \ldots, p\}$, а $S_{l} \cap S_{q}=0$ для $l \neq q$ и таких $p$ нормированных ( $D f^{(i)}=1$, кода дисперсия будет 1$)$ факторов $f^{(1)}, f^{(2)}, \ldots, f^{\left(p^{\prime}\right)}$. Они ведут к максимизации соответствующего критерия оптимальности.

По конкретному критерию оптимальности в спутниковых системах связи дадим анализ алгоритма.

Пусть функционал рассматривается как критерий оптимальности 


$$
J_{1}=\sum_{i \in S_{1}}\left[\operatorname{corr}\left(x^{(i)}, f^{(1)}\right)\right]^{2}+\ldots+\sum_{i \in S_{p^{\prime}}}\left[\operatorname{corr}\left(x^{(i)}, f^{p^{\prime}}\right)\right]^{2} .
$$

Для него $\operatorname{corr}(x, f)$ будет парным коэффициентом корреляции. Он связывает признак $x$ и фактор $f$ в спутниковой системе. Предположим, что $A_{i}=\left\{x^{(i)}, i \in S_{l}\right\}, l=1,2, \ldots, p^{\prime}$. Функционал $J_{1}$ максимизируется. Учитывается разбиение признаков по подмножествам $A_{1}, \ldots, A_{p}$. Еще учитывается выбор по факторам $\left.f^{(1)}, f^{(2)}, \ldots, f^{\left(p^{\prime}\right)}\right)$. В функционале [6], те признаки, которые «близки» между, будут размещаться внутри соответствующего подмножества. Тогда они будут высоким образом коррелированны. Это видно, поскольку когда $J_{1}$ будет максимизироваться, тогда в $l$-е подмножестве размещаются по фиксированному набору случайных величин $f^{(1)}, f^{(2)}, \ldots, f^{\left(p^{\prime}\right)}$. Для них можно говорить о сильной корреляции с величиной $f^{(l)}$. Но мы будем делать выбор по набору по возможным совокупностям случайных величин $f^{(1)}, f^{(2)}, \ldots, f^{\left(p^{\prime}\right)}$. Условием при этом будет «близость» каждой из величин $f^{(l)}$ относительно всех признаков соответствующего подмножества.

Получение оптимального набора факторов $f^{(1)}, f^{(2)}, \ldots, f^{\left(p^{\prime}\right)}$, если обозначены классы $S_{1}, S_{2}, \ldots S_{p}$, возможно как результат максимизации по каждому из слагаемых

$$
\sum_{i=S^{\prime}}\left[\operatorname{corr}\left(x^{(i)}, f^{(l)}\right)\right]^{2}\left(l=\overline{1, p^{\prime}}\right),
$$

Поэтому мы получаем $\max _{f^{(1)}, f^{(2)}, \ldots, f^{(l)}} J_{1}=\sum_{l=1}^{p} \lambda_{l}^{2}$.

В указанном выражении $\lambda_{l}$ - рассматривается в виде максимального собственного значения матрицы $R_{l}$. Формируется он при помощи коэффициентов корреляции переменных. Они являются компонентами $A_{l}$. Чтобы получить оптимальнное множество факторов $f^{(l)}, l=1,2, \ldots, p^{\prime}$, требуется провести расчет:

$$
f^{(l)}=\frac{\sum_{i \in S_{l}} \alpha_{i}^{(l)} x^{(i)}}{\sqrt{\sum_{i, j \in S_{l}} \alpha_{i}^{(l)} \alpha_{j}^{(l)} r_{j}}} l=1,2 \ldots, p^{\prime} .
$$


В указанном выражении $r_{j}=\operatorname{corr}\left(x^{(i)}, x^{(j)}\right)$, а $\alpha^{(l)}=$ $\left(\alpha_{1}^{(l)}, \alpha_{2}^{(l)}\right), \ldots, \alpha_{m_{l}}^{(l)}$ рассматривается в виде собственного вектора матрицы $R_{l}$. Он связан с собственным значением $\lambda_{l}$, т.е $R_{l} * \alpha^{(l)}=\lambda_{l} * \alpha^{(l)}$, являющимся максимальным.

Мы считаем, что факторы $f^{(1)}, f^{(2)}, \ldots, f^{\left(p^{\prime}\right)}$ рассматриваются в виде известных. Тогда мы можем разбить $S_{1}, S_{2}, \ldots S_{p}$, что позволит максимизировать $J_{1}$, когда фиксированы $f^{(1)}, f^{(2)}, \ldots, f^{\left(p^{\prime}\right)}$. Тогда

$$
S_{l}=\left\{i: \operatorname{corr}^{2}\left(x^{(i)}, f^{(i)}\right) \geq \operatorname{corr}^{2}\left(x^{(i)}, f^{(q)}\right) \text { для всех } q=1,2, \ldots ., p^{\prime}\right\},(3)
$$

Для того, чтобы был максимум $J_{1}$, можно рассматривать в виде необходимых условий (2) и (3).

Разбиения $S_{1}, S_{2}, \ldots S_{p}$ и множество факторов $f^{(1)}, f^{(2)}, \ldots, f^{\left(p^{\prime}\right)}$ должны быть в ходе поиска оптимальными. Тогда мы исходим из итерационного алгоритма. В нем выбираются оптимальные факторы. Они последовательным образом определяются. После этого выбирается разбиение, которое будет оптимальным относительно факторов, которые были получены для предыдущего шага [7].

Параметры соотносятся с подмножествами $A_{1}, \ldots, A_{p^{\prime}}$ для $\gamma$-м шага итерации. Проводим формирование факторов $f_{\gamma}^{\left.p^{\prime}\right)}$ на базе (2) в каждом из подмножества параметров. Также формируем $\gamma+1$ разбиение параметров $A_{1}^{(\gamma+1)}, \ldots, A_{p}^{(\gamma+1)}$. При этом исходим из правила: параметр $x^{(i)}$ связан с подмножеством $A_{l}^{(\gamma+1)}$, когда

$$
\operatorname{corr}^{2}\left(x^{(l)}, f_{\gamma}^{(l)}\right) \geq \operatorname{corr}^{2}\left(x^{(l)}, f_{\gamma}^{q}\right)\left(q=1,2, \ldots, p^{\prime}\right)
$$

Пусть мы сможем найти $\mathrm{n}>2$ факторов, когда придем к знаку равенства в (2). При выполнении такого условия принадлежность параметра $x^{(i)}$ к определенному множеству будет произвольной.

Не будет падения убывает $J_{1}$ для каждого из шагов итераций. Это определяет схождение к максимуму, в том числе и локальному, алгоритма. Произведен выбор набора показателей, основываясь на указанной методике, которые требуются для осуществления дифференциального анализа спутниковой системы связи [8].

Были сформированы такие подмножества показателей, являющихся взаимосвязанными:

1) число модулей спутниковой системы связи [9], 
2) ширина диаграммы направленности,

3) мощность передающего устройства,

4) коэффициент направленного действия.

Использование деревьев решений для того, чтобы формировать модели анализа. По спутниковой системе связи важно сформировать модель дифференциального анализа. Применяем методологию, связанную с построением «деревьев решений» [10].

Применяем в ходе исследований линейную решающую функцию, как элементарную функцию

$$
g(X)=\bar{W}^{\mathrm{T}} \bar{X}+\omega_{n+1} .
$$

В указанном выражении $\bar{X}$ рассматривается в виде вектора признаков, $\bar{X}=\left(x_{1}, x_{2}, \ldots, x_{n}\right)$,

$\bar{W}_{\text {。 }}$ рассматривается в виде весового вектора, $\bar{W}_{0}=\left(\omega_{1}, \omega_{2}, \ldots, \omega_{n}\right)$.

Пусть ведем анализ расширенных векторов признаков. При этом они имеют дополнительную последнюю компоненту 1 . В таком случае мы запишем соотношение:

$$
g(X)=W^{\prime} X
$$

в нем $X=\left(x_{l}, x_{2}, \ldots, x_{n^{\prime}}, 1\right), W=\left(\omega_{1}, \omega_{2}, \ldots, \omega_{n+1}\right)$.

Исходим из того, что, что когда разбиваем по 2 классам $\omega_{1}, \omega_{2}$, тогда для решающей функции имеем условия

$$
g(X)=W^{\prime} X \begin{cases}>0 & \text { е с ли } X \in \omega_{1}, \\ <0 & \text { е сли } X \in \omega_{2} .\end{cases}
$$

Число классов $\omega_{1}, \omega_{2}, \ldots, \omega_{n}$, может быть в ходе исследований и более, чем 2.

Метод попарного разделения по классам может рассматриваться в виде наиболее общего подхода для указанных типов задач.

Тогда ведем отделение одного класс от другого за счет того, что есть индивидуальная разделяющая поверхность. Считаем, что есть попарная разделимость в классах. Мы можем найти $C_{M}^{2}=\frac{M(M-1)}{2}$ поверхностей, являющихся разделяющими. Для решающих функций представление такое: $g_{i j}(X)=W_{i j}^{\prime} X$. Для них характерно, что когда образ $\bar{X}$ будет принадлежать к классу $\omega_{i}$, тогда $g_{i j}(X)>0$, при условии $j \neq i$. Также, $g_{i j}(X)=-g_{j i}(X)$. 
Требуются высокие вычислительные затраты, чтобы осуществить процесс попарного разделения по полному множеству решающих функций $\frac{M(M-1)}{2}$.

Большое число попарным образом разделяющих функций будут дублировать друг друга. Это демонстрирует практика. Такие дублирующие функции мы можем убрать. Тогда для окончательного решающего правила мы приходим к дереву решений. Для него мы выбираем линейные решающие функции последовательным образом. Они будут удовлетворять соответствующим критериям.

\section{Выводы}

В проведенном исследовании даны предложения по возможностям построения алгоритма для анализа параметров в спутниковой системе связи. Комбинируя его с подходом, базирующимся на использовании деревьев решений, есть возможности для того, чтобы повысить эффективность обработки данных в спутниковых системах связи.

Информация о конфликте интересов. Авторы заявляют об отсутствии конфликта интересов.

Информация о спонсорстве. Исследование не имело спонсорской поддержки.

\section{Список литературы}

1. Суворов А.П., Лесников А.С. Особенности развития современных телекоммуникационных сетей // Вестник Воронежского института высоких технологий. 2020. № 1 (32). С. 46-48.

2. Львович И.Я., Кравцова Н.Е., Чупринская Ю.Л. Об использовании подходов базирующихся на технологии data mining // Вестник Воронежского института высоких технологий. 2020. № 1 (32). С. 28-30.

3. Преображенский Ю.П., Чопоров О.Н. Об использовании метода статистического анализа для анализа характеристик производительности сети // Вестник Воронежского института высоких технологий. 2020. № 2 (33). С. 34-36. 
4. Львович Э.М., Холодков А.М. Проблемы передачи информации в автоматизированных системах управления // Вестник Воронежского института высоких технологий. 2020. № 3 (34). C. 30-33.

5. Преображенский Ю.П., Чупринская Ю.Л., Кравцова Н.Е. Анализ характеристик, используемых при проектировании беспроводных систем связи // Вестник Воронежского института высоких технологий. 2020. № 3 (34). С. 47-49.

6. Щетникова Д.А., Маренков Н.М., Ружицкий Е. Проблемы моделирования распространения радиоволн на базе лучевых подходов // Вестник Воронежского института высоких технологий. 2020. № 3 (34). С. 56-58.

7. Львович И.Я., Чупринская Ю.Л., Кравцова Н.Е. О разделении и анализе перекрещивающихся сигналов // Вестник Воронежского института высоких технологий. 2020. № 4 (35). С. 65-67.

8. Бабешко В.Н., Черняков М.К., Чернякова М.М. Оценка эвристических методов системного анализа // International Journal of Advanced Studies. 2018. T. 8, № 1-2. C. 15-20.

9. Лысанов Д.М., Пономаренко Н.А. Количественная оценка уровня качества оборудования // International Journal of Advanced Studies. 2018. T. 8, № 4-2. С. 56-61.

10.Волков Д.В. Имитационное мультиагентное моделирование системы связи специального назначения // International Journal of Advanced Studies. 2017. T. 7, № 1-2. C. 31-37.

\section{References}

1. Suvorov A.P., Lesnikov A.S. Vestnik Voronezhskogo instituta vysokih tekhnologij, 2020, no. 1 (32), pp. 46-48.

2. L'vovich I.YA., Kravcova N.E., Chuprinskaya Yu.L. Vestnik Voronezhskogo instituta vysokih tekhnologij, 2020, no. 1 (32), pp. 28-30.

3. Preobrazhenskij Yu.P., CHoporov O.N. Vestnik Voronezhskogo instituta vysokih tekhnologij, 2020, no. 2 (33), pp. 34-36.

4. L'vovich E.M., Holodkov A.M. Vestnik Voronezhskogo instituta vysokih tekhnologij, 2020, no. 3 (34), pp. 30-33. 
5. Preobrazhenskij Yu.P., CHuprinskaya Yu.L., Kravcova N.E. Vestnik Voronezhskogo instituta vysokih tekhnologij, 2020, no. 3 (34), pp. 47-49.

6. Shchetnikova D.A., Marenkov N.M., Ruzhickij E. Vestnik Voronezhskogo instituta vysokih tekhnologij, 2020, no. 3 (34), pp. 56-58.

7. L'vovich I.Ya., CHuprinskaya Yu.L., Kravcova N.E. Vestnik Voronezhskogo instituta vysokih tekhnologij, 2020, no. 4 (35), pp. 65-67.

8. Babeshko V.N., Chernyakov M.K., CHernyakova M.M. International Journal of Advanced Studies, 2018, vol. 8, no. 1-2, pp. 15-20.

9. Lysanov D.M., Ponomarenko N.A. International Journal of Advanced Studies, 2018, vol. 8, no. 4-2, pp. 56-61.

10. Volkov D.V. International Journal of Advanced Studies, 2017, vol. 7, no. 1-2, pp. 31-37.

\section{ДАННЫЕ ОБ АВТОРАХ \\ Мельникова Томара Вениаминовна, магистр}

Автономная некоммерческая образовательная организация высшего образования Воронежский институт высоких технологий

ул. Ленина, 73а, г. Воронеж, 394043, Российская Федерация tmelnikiva910@gmail.com

Преображенский Андрей Петрович, профессор, доктор технических наук, доцент Автономная некоммерческая образовательная организаџия высшего образования Воронежский институт высоких технологий

ул. Ленина, 73а, г. Воронеж, 394043, Российская Федераиия Komkovvivt@yandex.ru

Преображенский Юрий Петрович, профессор, кандидат технических наук, доцент Автономная некоммерческая образовательная организация высшего образования Воронежский институт высоких технологий 
ул. Ленина, 73а, г. Воронеж, 394043, Российская Федерация Komkovvivt@yandex.ru

\section{DATA ABOUT THE AUTHORS}

Tomara V. Melnikova, Magistr

Voronezh Institute of High Technologies

73a, Lenin Str., Voronezh, 394043, Russian Federation tmelnikiva910@gmail.com

Andrey P. Preobrazhenskiy, Doctor of Technical Sciences, Associate Professor

Voronezh Institute of High Technologies

73a, Lenin Str., Voronezh, 394043, Russian Federation

Komkovvivt@yandex.ru

ORCID: 0000-0002-6911-8053

Yuriy P. Preobrazhenskiy, Professor, Candidate of Technical Sciences, Associate Professor Voronezh Institute of High Technologies

73a, Lenin Str., Voronezh, 394043, Russian Federation Komkovvivt@yandex.ru 